\begin{tabular}{|c|c|}
\hline Title & $\begin{array}{l}\text { Nonlinear propagation of a few-optical-cycle pulses in a photonic crystal fiber-experimental and theoretical studies } \\
\text { bey ond the slowly varying-envelope approximation }\end{array}$ \\
\hline Author(s) & Fang, Xiaojun; Karasawa, Naoki; Morita, Ryuji; Windeler, Robert S.; Y amashita, Mikio \\
\hline Citation & $\begin{array}{l}\text { IEEE Photonics Technology Letters, 15(2), } 233-235 \\
\text { https://doi.org/10.1109/LPT.2002.806082 }\end{array}$ \\
\hline Issue Date & $2003-02$ \\
\hline Doc URL & http:/hdl .handle.net/2115/45336 \\
\hline Rights & $\begin{array}{l}\text { ( } 2003 \text { IEEE. Personal use of this material is permitted. However, permission to reprint/republish this material for } \\
\text { advertising or promotional purposes or for creating new collective works for resale or redistribution to servers or lists, } \\
\text { or to reuse any copyrighted component of this work in other works must be obtained from the IEEE. }\end{array}$ \\
\hline Type & article \\
\hline File Information & PTL15-2_233-235.pdf \\
\hline
\end{tabular}

Instructions for use 


\title{
Nonlinear Propagation of a-Few-Optical-Cycle Pulses in a Photonic Crystal Fiber- Experimental and Theoretical Studies Beyond the Slowly Varying-Envelope Approximation
}

\author{
Xiaojun Fang, Naoki Karasawa, Ryuji Morita, Robert S. Windeler, and Mikio Yamashita
}

\begin{abstract}
The evolution of spectral and temporal profiles of 4.5 optical-cycle pulses propagating near zero-dispersion wavelength (ZDW) in a photonic crystal fiber is investigated experimentally and theoretically beyond the slowly varying-envelope approximation. The excellent agreement between the experimental and theoretical results suggests that the observed gap in the spectral profile, the most distinctive feature, originates from the self-steepening effect. This effect intensifies the spectral component shorter than the ZDW with the decay of higher order solitons and consequently induces the intrapulse four-wave mixing (FWM). As a result, the anti-Stokes and Stokes components produced by the FWM enables us to generate a supercontinuum from 480 to $1020 \mathrm{~nm}$.
\end{abstract}

Index Terms-A-few-cycle pulse propagation, four-wave mixing (FWM), optical solitons supercontinuum generation, photonic crystal fiber, self steepening (SST), slowly varying envelope approximation (SVEA) free.

\section{INTRODUCTION}

$\mathbf{P}$ HOTONIC crystal fibers (PCFs) have attracted significant research attention in recent years for their zero-dispersion wavelength (ZDW) shifted into the visible range and hence efficient supercontinuum (SC) generation [1], [2]. However, the mechanism in SC generation is not yet well understood due to the complicated interactions among many nonlinear optical processes such as self-phase modulation (SPM), stimulated Raman scattering (SRS), parametric four-wave mixing (FWM), soliton formation, and self-steepening (SST), as well as the unusual dispersion profile. As for the parametric FWM in a PCF, there are two types, conventional FWM and intrapulse FWM. Conventional FWM is based on the third-order nonlinear polarization induced by the interaction among optical electric fields with different frequency components. That is, one pump wave creates two new waves with different frequency bands from the spontaneous-emission noise outside the pump-pulse spectral band only if the phase matching condition is satisfied. On the other hand, intrapulse FWM describes the effective SPM on the basis

Manuscript received April 8, 2002; revised September 4, 2002.

X. Fang, R. Morita, and M. Yamashita are with the Department of Applied Physics, Hokkaido University, and CREST.JST, Sapporo 060-8628, Japan (e-mail: fang@eng.hokudai.ac.jp).

N. Karasawa is with the Department of Applied Photonics Technology System, Chitose Institute of Science and Technology, Chitase 066-8, Japan.

R. S. Windeler is with Bell Laboratories, Lucent Technologies, Murray Hill, NJ 07974 USA

Digital Object Identifier 10.1109/LPT.2002.806082 of the third-order nonlinear polarization induced by the interaction among electric fields with the same frequency components, from the point of view of the frequency domain. That is, certain spectral bands inside the broad-band spectrum of one pulse are selected and grown as a result of mixing of intrapulse different-frequency components satisfying phase matching and others cannot be frequency mixed in an efficient way. In this letter, we take notice of intrapulse FWM.

In 2000, the authors of [1] first demonstrated that the combined effects of SPM and SRS in the long length of the fiber $(75 \mathrm{~cm})$ produced SC from 390 to $1600 \mathrm{~nm}$, where 100-fs input pulses with 8-kW peak power and a center wavelength $(790 \mathrm{~nm})$ slightly longer than ZDW (767 nm) were propagated. In 2001, another group [2] reported that the experimentally generated SC from 400 to $1000 \mathrm{~nm}$ with two spectrally sharp peaks near the center wavelength was due to the combined effects of SRS and conventional FWM with a negligible contribution from SPM in the longer length of the fiber $(10 \mathrm{~m})$, where 60 -ps pulses with $675-W$ peak power and a center wavelength $(647 \mathrm{~nm})$ slightly shorter than ZDW $(675 \mathrm{~nm})$ were propagated. More recently, by a theoretical analysis for optical pulse propagation in the short-length PCF $(7.5 \mathrm{~cm})$, it has been shown that the SC generation from 400 to $1000 \mathrm{~nm}$ is due to the fission of high-order solitons into red-shifted fundamental solitons and blue-shifted nonsolitonic radiation, where 100 -fs input pulses with severalkilowatt peak power and a center wavelength $(830 \mathrm{~nm})$ longer than ZDW $(710 \mathrm{~nm})$ are propagated [3]. Furthermore, numerical analysis has indicated that 17.5 -fs short-pulse propagation does not cause the dramatic spectral broadening. In its analysis, however, the employed equation does not consider the effect of the delayed Raman response, which has characteristic constants of several-ten femtoseconds [4], and in general is not valid for propagation of single optical-cycle pulses and ultrabroad-band pulses exceeding an octave bandwidth [4], [5].

The purpose of this letter is to clarify both experimentally and theoretically the mechanism in SC generation for propagation of a-few-cycle pulses in a PCF by obtaining systematic information on spectral and temporal evolutions of the pulses at different propagation distances.

With ZDW at $767 \mathrm{~nm}$ and negative group-delay dispersion (GDD) at its longer wavelength, high-delta PCFs used in our experiment consist of a $1.7-\mu \mathrm{m}$-diameter undoped silica core surrounded by an array of $1.3-\mu \mathrm{m}$-diameter air holes in a hexagonal close-packed arrangement [1]. 12-fs-duration pulses with $800-\mathrm{mW}$ average power centered $795 \mathrm{~nm}$ were generated 

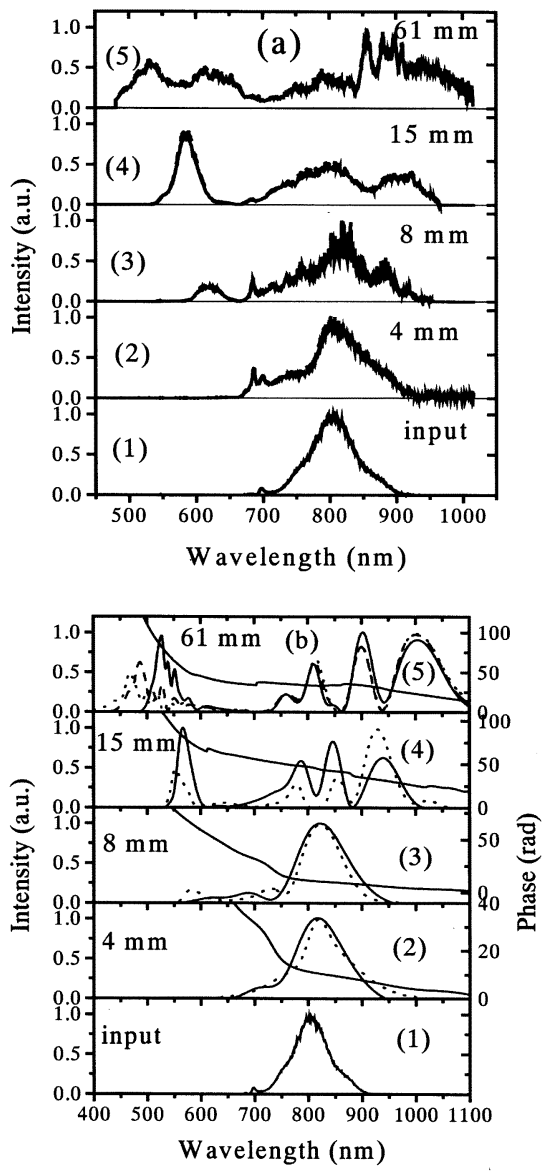

Fig. 1. (a) Experimental spectra generated from 4.5-cycle optical pulses in different-length PCFs. (b) Numerically simulated intensity and phase spectra with SEWA (solid lines), SVEA (dotted lines), and SVEA plus all-order dispersion (dashed lines).

from a mode-locked $\mathrm{Ti}$ : sapphire laser at the repetition rate of $75 \mathrm{MHz}$. The corresponding spectrum ranged from 700 to $910 \mathrm{~nm}$ with $69-\mathrm{nm}$ width (full-width at half maximum), as shown in Fig. 1(a)-(1).

PCFs with different lengths $(4,8,15,61 \mathrm{~mm})$ were carefully cleaved to produce both pristine and undamaged surface. Pulses were then coupled into those PCFs by a reflective objective of $36 \times$ magnification to avoid additional dispersion from transmission in a dielectric medium. The fibers were rotated to make the polarization axis of the linearly polarized pulses coincide with one of the principal axes in the fibers. Outputs from the fibers were collimated by another identical reflective objective. The spectra of the pulses before and after propagation [Fig. 1(a)] were measured by a calibrated spectrometer. In addition, their corresponding pulse durations [the insets of Fig. 2(a)] were characterized by an intensity autocorrelator. The typical input and output average powers were 400 and $60 \mathrm{~mW}$, respectively.

From Fig. 1(a) we find that the evolution of the intensity spectra during propagation has the following notable features. At first $(4 \mathrm{~mm})$, the spectrum broadens asymmetrically (625 to $915 \mathrm{~nm}$ ) to form a shoulder on the blue side. With an increase in propagation distance $(8 \mathrm{~mm})$, the asymmetric spectrum continues to broaden (590 to $950 \mathrm{~nm}$ ) with small peaks at the blue $(625 \mathrm{~nm})$ and red $(915 \mathrm{~nm})$ edges and the spectral gap appears

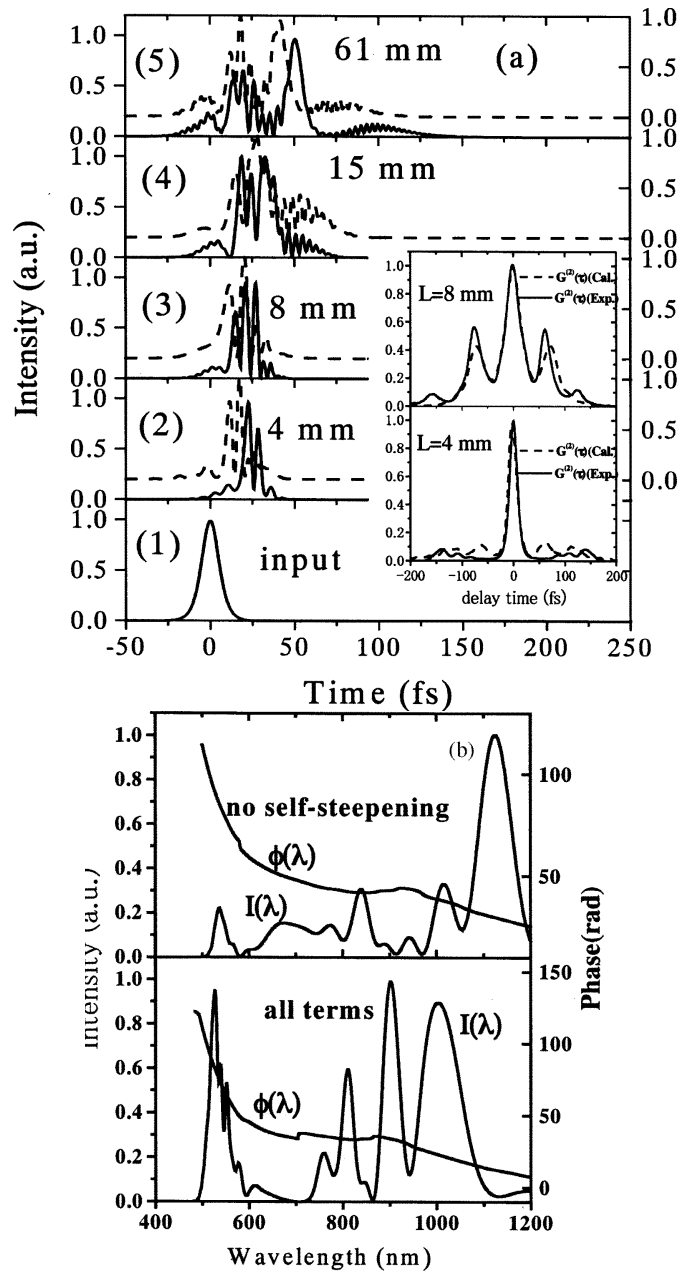

Fig. 2. (a) Numerically simulated temporal-intensity profiles with SEWA (solid lines) and with SVEA (dashed lines). The insets are the experimental intensity autocorrelation traces (solid lines) with the corresponding calculated results (dashed lines) with increasing propagation distance. (b) Effect of the self-steepening in spectral broadening.

near $660 \mathrm{~nm}$. After the $15-\mathrm{mm}$ distance with the further asymmetric broadening, the peak at the blue edge $(580 \mathrm{~nm})$ grows in size and the red edge extends to $965 \mathrm{~nm}$. In addition, the spectral gap $(650 \mathrm{~nm})$ becomes more clear. Finally (the $61-\mathrm{mm}$ distance), the spectrum broadens the most (480-1020 nm) with two peaks on the blue side (530 and $610 \mathrm{~nm}$ ) and several peaks on the red side.

To understand the mechanism of the experimentally generated SC, we employ a theoretical equation beyond the slowly varying-envelope approximation (SVEA), which is based on an extension [6] of the generic nonlinear envelope equation (NEE) of the first-order in the propagation coordinate. The NEE was derived from Maxwell's wave equation by effectively taking into account the second derivative in retarded time $T$ in the group-velocity coordinate and the first derivative in position $\xi$ under the condition of a small difference between the group and phase velocities. This is called the slowly evolving wave approximation (SEWA) [5]. Its validity for the description of optical pulse propagation down to the single-cycle region was confirmed for a conventional glass fiber [5], [6]. The extended NEE [6] includes the contribution of the delayed Raman response to nonlinear polarization and the exact SST term, besides the all 
orders of terms for effective linear dispersion of a PCF [1]. That is, the extended NEE [6, eq. (12)] for the complex amplitude of the pulse electric field $A(\xi, T)$ is numerically solved by the split-step method [7] using input pulse and fiber parameters satisfying the experimental conditions (the same notations as those in [6])

The calculated evolution of the spectral and temporal profiles as a function of the propagation distance is shown in Fig. 1(b) and 2(a). The agreement between the experiment and the calculation is excellent.

The conventional equation in the SVEA case with inclusion of the SST effect and the delayed-nonlinear Raman effect is different from (1), as follows: 1) up to the third-order linear dispersion (TOD) for $\hat{D} ; 2)$ no $\hat{D}_{\text {corr }}$ term; and 3 ) the approximate SST coefficient of $s^{\prime}=1 / \omega_{0},\left(\omega_{0}\right.$ : the center angular frequency of the input pulse). The calculated result indicated that the spectral broadening at shorter wavelength is much larger than compared with the experimental result, especially at the longest propagation distance, as shown by dotted lines of Fig. 1(b)-(1) (5). Furthermore, we find that even if we take account of rigorous linear dispersion terms, the spectral broadening at the short wavelength is still larger, as shown by a dashed line for the longest distance. The difference is mainly due to neglect of the $\hat{D}_{\text {corr }}$ term rather than the approximate SST coefficient, unlike the conventional glass-fiber case [6]. The $\hat{D}_{\text {corr }}$ term, which results from no neglect of the second derivative in retarded time, describes the effective ratio between the square of the sum of the higher order linear dispersions than the GDD and the sum of up to the first-order linear dispersion in the frequency domain, and varies largely in the shorter wavelength region than around $600 \mathrm{~nm}$ (especially the effective TOD variation) for the present PCF. Consequently, the $\hat{D}_{\text {corr }}$ term provides the excellent agreement between the theory and the experiment concerning a relationship between the spectral-broadening width after long propagation and the propagation distance.

From Figs. 1 and 2, the mechanism in SC generation for PCF propagation of a-few-cycle pulses with several-ten-kilowatt peak power $(67 \mathrm{~kW}$ : the soliton number $N=5.7)$ and a central wavelength $(795 \mathrm{~nm})$ slightly longer than ZDW $(767 \mathrm{~nm})$ is found out as follows. At first (up to several millimeters), the combined effects of SPM and negative group-velocity dispersion play a main role (the nonlinear length of $L_{N L}=0.2 \mathrm{~mm}$ and the dispersion lengths of the second-order $L_{D}=18 \mathrm{~mm}$ and the third-order $L_{D}^{\prime}=21 \mathrm{~mm}$ ). That is, as the input-spectral peak lies in the negative GDD region, most of the pulse energy in the spectral band forms a higher order soliton $(N=5.7$ and the ZDW soliton number $\tilde{N}=4.7$ [7]). Since the critical parameter of $\delta_{3}=0.24$ [7] representing the relative effect of the TOD to the group-velocity dispersion on soliton formation is considerably large, asymmetric multifold splitting of the temporal-intensity profile occurs [7]. In addition, the SST effect gradually influences soliton propagation (the SST critical distance of $L_{s}=2.3 \mathrm{~mm}$ ) so that the spectrum is blue-shifted with asymmetric broadening [(2) of Fig. 1], and the temporal intensity is steepened on the trailing side [(2) of Fig. 2(a)]. The combined effects of TOD and SST slow down higher order solitions and hence the splitting pulse is delayed [(2) of Fig. 2(a)]. Around the 8-mm propagation distance [(3) of Fig. 1], the SST- induced short-wavelength component in the positive GDD region $(<767 \mathrm{~nm})$ efficiently causes the intrapulse FWM. This is because the intensified wavelength component, slightly shorter than ZDW, plays the role of the pump $\left(\omega_{p}\right)$ for the FWM processes [for example, $\omega_{p}(766 \mathrm{~nm})+\omega_{p}(766 \mathrm{~nm}) \rightarrow \omega_{s}(930 \mathrm{~nm})$ $\left.+\omega_{a s}(610 \mathrm{~nm})\right]$, and phase matches intrinsically with intrapulse Stokes $\left(\omega_{s}\right)$ and anti-Stokes $\left(\omega_{a s}\right)$ components [2], as well as having the nearly group-velocity matching and the parametric gain. As a result, the anti-Stokes and Stokes wavelength components appear and the spectral gap is yielded. The further propagation [(4) of Fig. 1] extends their wavelength regions to try the phase matching. However, the degree of the wavelength-dependent group-velocity matching worsens and the wavelengthdependent parametric gain is reduced [2], [7]. The result of spectral broadening gives rise to the temporal pulse broadening to form the wings because of the large dispersion in their regions. In addition, the large TOD causes the oscillatory structure near the trailing edge of the pulse. Finally [Fig. 1 (5)], the intensified anti-Stokes and Stokes waves are spectrally modulated due to the wavelength-dependent group-velocity matching and parametric gain, with reduction of the spectral-broadening speed of the anti-Stokes component by the $\hat{D}_{\text {corr }}$ effect. Consequently, the ultrabroad-band pulse from 480 to $1020 \mathrm{~nm}$ is generated. These findings suggest that the SST effect, intensifying the spectral component shorter than ZDW with the decay of higher order solitons and hence causing the intrapulse FWM, is essential for the existence of the spectral gap. This spectral behavior is the most striking feature appearing in propagation of a-few-cycle pulses with a center wavelength slightly longer than ZDW in a PCF. Fig. 2(b), indicating the comparison between the calculated results without and with the SST term, gives evidence for this point. We also confirmed that neglect of the delayed Raman response worsens the agreement with the experimental result for the propagation evolution of the whole spectral profile, especially for the spectral shift of the anti-Stokes component.

In conclusion, the evolution of the SC, generated by $\mathrm{PCF}$ propagation of a-few-cycle pulses, has been well explained by a numerical analysis based on the extended NEE beyond the SVEA.

\section{REFERENCES}

[1] J. Ranka, R. S. Windler, and A. J. Stentz, "Visible continuum generation in air-silica microstructure optical fibers with anomalous dispersion at 800 nm," Opt. Lett., vol. 25, pp. 25-27, 2000.

[2] S. Coen, A. Hing, L. Chau, R. Leonhardt, J. D. Harvey, J. C. Knight, W. J. Wadsworth, and P. St. J. Russell, "White-light supercontinuum generation with 60-ps pump pulse in a photonic crystal fiber," Opt. Lett., vol. 26, pp. 1356-1358, 2001.

[3] A. V. Husakou and J. Herrmann, "Supercontinuum generation of higherorder solitons by fission in photonic crystal fibers," Phys. Rev. Lett., vol. 87, pp. 203901-1-203 901-4, 2001.

[4] K. J. Blow and D. Wood, "Theoretical description of transient stimulated Raman scattering in optical fibers," IEEE J. Quantum Electron., vol. 25, pp. 2665-2673, Dec. 1989.

[5] T. Brabec and F. Krausz, "Nonlinear optical pulse propagation in the single-cycle regime," Phys. Rev. Lett., vol. 78, pp. 3282-3285, 1997.

[6] N. Karasawa, S. Nakamura, N. Nakagawa, M. Shibata, R. Morita, H. Shigekawa, and M. Yamashita, "Comparison between theory and experiment of nonlinear propagation for a-few-cycle and ultrabroadband optical pulses in a fused-silica fiber," IEEE J. Quantum Electron., vol. 37, pp. 398-404, Mar. 2001.

[7] G. P. Agrawal, Nonlinear Fiber Optics, 3rd ed. San Diego, CA: Academic, 2001, ch. 2, 5, and 10. 